\title{
AN INFINITE C*-ALGEBRA WITH A DENSE, STABLY FINITE *-SUBALGEBRA
}

\author{
NIELS JAKOB LAUSTSEN AND JARED T. WHITE
}

\begin{abstract}
We construct a unital pre- $\mathrm{C}^{*}$-algebra $A_{0}$ which is stably finite, in the sense that every left invertible square matrix over $A_{0}$ is right invertible, while the $\mathrm{C}^{*}$-completion of $A_{0}$ contains a non-unitary isometry, and so it is infinite.
\end{abstract}

To appear in Proceedings of the American Mathematical Society.

\section{INTRODUCTION}

Let $A$ be a unital algebra. We say that $A$ is finite (also called directly finite or Dedekind finite) if every left invertible element of $A$ is right invertible, and we say that $A$ is infinite otherwise. This notion originates in the seminal studies of projections in von Neumann algebras carried out by Murray and von Neumann in the 1930s. At the $22^{\text {nd }}$ International Conference on Banach Algebras and Applications, held at the Fields Institute in Toronto in 2015, Yemon Choi raised the following questions:

(1) Let $A$ be a unital, finite normed algebra. Must its completion be finite?

(2) Let $A$ be a unital, finite pre- $\mathrm{C}^{*}$-algebra. Must its completion be finite?

Choi also stated Question (1) in [7, Section 6].

A unital algebra $A$ is said to be stably finite if the matrix algebra $M_{n}(A)$ is finite for each $n \in \mathbb{N}$. This stronger form of finiteness is particularly useful in the context of $K$ theory, and so it has become a household item in the Elliott classification programme for $\mathrm{C}^{*}$-algebras. The notions of finiteness and stable finiteness differ even for $\mathrm{C}^{*}$-algebras, as was shown independently by Clarke [8] and Blackadar [4] (or see [5, Exercise 6.10.1]). A much deeper result is due to Rørdam [9, Corollary 7.2], who constructed a unital, simple $\mathrm{C}^{*}$-algebra which is finite (and separable and nuclear), but not stably finite.

We shall answer Question (2), and hence Question (1), in the negative by proving the following result:

Theorem 1.1. There exists a unital, infinite $C^{*}$-algebra which contains a dense, unital, stably finite ${ }^{*}$-subalgebra.

Let $A$ be a unital *-algebra. Then there is a natural variant of finiteness in this setting, namely we say that $A$ is ${ }^{*}$-finite if whenever we have $u \in A$ satisfying $u^{*} u=1$, then $u u^{*}=1$. However, it is known (see, e.g., [10, Lemma 5.1.2]) that a $\mathrm{C}^{*}$-algebra is finite if and only if it is ${ }^{*}$-finite, so in this article we shall not need to refer to ${ }^{*}$-finiteness again.

2010 Mathematics Subject Classification. 46L05 (primary); 20M25, 46L09 (secondary).

Key words and phrases. C*-algebra, stably finite, infinite, completion, free product. 


\section{Preliminaries}

Our approach is based on semigroup algebras. Let $S$ be a monoid, that is, a semigroup with an identity, which we shall usually denote by $e$. By an involution on $S$ we mean a map from $S$ to $S$, always denoted by $s \mapsto s^{*}$, satisfying $(s t)^{*}=t^{*} s^{*}$ and $s^{* *}=s(s, t \in S)$. By a ${ }^{*}$-monoid we shall mean a pair $(S, *)$, where $S$ is a monoid, and $*$ is an involution on $S$. Given a ${ }^{*}$-monoid $S$, the semigroup algebra $\mathbb{C} S$ becomes a unital *-algebra simply by defining $\delta_{s}^{*}=\delta_{s^{*}}(s \in S)$, and extending conjugate-linearly.

Next we shall recall some basic facts about free products of *-monoids, unital *-algebras, and their $\mathrm{C}^{*}$-representations.

Let $S$ and $T$ be monoids, and let $A$ and $B$ be unital algebras. Then we denote the free product (i.e. the coproduct) of $S$ and $T$ in the category of monoids by $S * T$, and similarly we denote the free product of the unital algebras $A$ and $B$ by $A * B$. It follows from the universal property satisfied by free products that, for monoids $S$ and $T$, we have $\mathbb{C}(S * T) \cong(\mathbb{C} S) *(\mathbb{C} T)$.

Given *-monoids $S$ and $T$, we can define an involution on $S * T$ by

$$
\left(s_{1} t_{1} \cdots s_{n} t_{n}\right)^{*}=t_{n}^{*} s_{n}^{*} \cdots t_{1}^{*} s_{1}^{*}
$$

for $n \in \mathbb{N}, s_{1} \in S, s_{2}, \ldots, s_{n} \in S \backslash\{e\}, t_{1}, \ldots, t_{n-1} \in T \backslash\{e\}$, and $t_{n} \in T$. The resulting *monoid, which we continue to denote by $S * T$, is the free product in the category of *-monoids. We can analogously define an involution on the free product of two unital *-algebras, and again the result is the free product in the category of unital *-algebras. We then find that $\mathbb{C}(S * T) \cong(\mathbb{C} S) *(\mathbb{C} T)$ as unital *-algebras.

Let $A$ be a ${ }^{*}$-algebra. If there exists an injective *-homomorphism from $A$ into some $\mathrm{C}^{*}$ algebra, then we say that $A$ admits a faithful $C^{*}$-representation. In this case, $A$ admits a norm such that the completion of $A$ in this norm is a $\mathrm{C}^{*}$-algebra, and we say that $A$ admits a $C^{*}$-completion. Our construction will be based on $\mathrm{C}^{*}$-completions of *-algebras of the form $\mathbb{C} S$, for $S$ a ${ }^{*}$-monoid.

We shall denote by $S_{\infty}$ the free *-monoid on countably many generators; that is, as a monoid $S_{\infty}$ is free on some countably-infinite generating set $\left\{t_{n}, s_{n}: n \in \mathbb{N}\right\}$, and the involution is determined by $t_{n}^{*}=s_{n}(n \in \mathbb{N})$. For the rest of the text we shall simply write $t_{n}^{*}$ in place of $s_{n}$. We define $B C$ to be the bicyclic monoid $\langle p, q: p q=e\rangle$. This becomes a ${ }^{*}$-monoid when an involution is defined by $p^{*}=q$, and the corresponding $*$-algebra $\mathbb{C} B C$ is infinite because $\delta_{p} \delta_{q}=\delta_{e}$, but $\delta_{q} \delta_{p}=\delta_{q p} \neq \delta_{e}$.

Lemma 2.1. The following unital ${ }^{*}$-algebras admit faithful $C^{*}$-representations:

(i) $\mathbb{C}(B C)$,

(ii) $\mathbb{C}\left(S_{\infty}\right)$.

Proof. (i) Since $B C$ is an inverse semigroup, this follows from [2, Theorem 2.3].

(ii) By [3, Theorem 3.4] $\mathbb{C} S_{2}$ admits a faithful $\mathrm{C}^{*}$-representation, where $S_{2}$ denotes the free monoid on two generators $S_{2}=\langle a, b\rangle$, endowed with the involution determined by $a^{*}=b$. There is a ${ }^{*}$-monomorphism $S_{\infty} \hookrightarrow S_{2}$ defined by $t_{n} \mapsto a\left(a^{*}\right)^{n} a(n \in \mathbb{N})$ and this induces a *-monomorphism $\mathbb{C} S_{\infty} \hookrightarrow \mathbb{C} S_{2}$. The result follows.

By a state on a unital *-algebra $A$ we mean a linear functional $\mu: A \rightarrow \mathbb{C}$ satisfying $\left\langle a^{*} a, \mu\right\rangle \geq 0(a \in A)$ and $\langle 1, \mu\rangle=1$. We say that a state $\mu$ is faithful if $\left\langle a^{*} a, \mu\right\rangle>0(a \in$ $A \backslash\{0\})$. A unital *-algebra with a faithful state admits a faithful $\mathrm{C}^{*}$-representation via the GNS representation associated with the state. 
The following theorem appears to be folklore in the theory of free products of $C^{*}$-algebras; it can be traced back at least to the seminal work of Avitzour [1, Proposition 2.3] (see also [6, Section 4] for a more general result).

Theorem 2.2. Let $A$ and $B$ be unital $*$-algebras which admit faithful states. Then their free product $A * B$ also admits a faithful state, and hence it has a faithful $C^{*}$-representation.

We make use of this result in our next lemma.

Lemma 2.3. The unital ${ }^{*}$-algebra $\mathbb{C}\left(B C * S_{\infty}\right)$ admits a faithful $C^{*}$-representation.

Proof. We first remark that a separable $\mathrm{C}^{*}$-algebra $A$ always admits a faithful state. To see this, note that the unit ball of $A^{*}$ with the weak*-topology is a compact metric space, and hence also separable. It follows that the set of states $S(A)$ is weak*-separable. Taking $\left\{\rho_{n}: n \in \mathbb{N}\right\}$ to be a dense subset of $S(A)$, we then define $\rho=\sum_{n=1}^{\infty} 2^{-n} \rho_{n}$, which is easily seen to be a faithful state on $A$.

By Lemma 2.1, both $\mathbb{C}(B C)$ and $\mathbb{C}\left(S_{\infty}\right)$ admit $\mathrm{C}^{*}$-completions. Since both of these algebras have countable dimension, their $\mathrm{C}^{*}$-completions are separable, and, as such, each admits a faithful state, which we may then restrict to obtain faithful states on $\mathbb{C} B C$ and $\mathbb{C} S_{\infty}$. By Theorem $2.2,(\mathbb{C} B C) *\left(\mathbb{C} S_{\infty}\right) \cong \mathbb{C}\left(B C * S_{\infty}\right)$ admits a faithful $\mathrm{C}^{*}$-representation.

\section{Proof of Theorem 1.1}

The main idea of the proof is to embed $\mathbb{C} S_{\infty}$, which is finite, as a dense ${ }^{*}$-subalgebra of some $\mathrm{C}^{*}$-completion of $\mathbb{C}\left(B C * S_{\infty}\right)$, which will necessarily be infinite. In fact we have the following:

Lemma 3.1. The ${ }^{*}$-algebra $\mathbb{C} S_{\infty}$ is stably finite.

Proof. As we remarked in the proof of Lemma 2.1, $\mathbb{C} S_{\infty}$ embeds into $\mathbb{C} S_{2}$. It is also clear that, as an algebra, $\mathbb{C} S_{2}$ embeds into $\mathbb{C} F_{2}$, where $F_{2}$ denotes the free group on two generators. Hence $\mathbb{C} S_{\infty}$ embeds into vN $\left(F_{2}\right)$, the group von Neumann algebra of $F_{2}$, which is stably finite since it is a $\mathrm{C}^{*}$-algebra with a faithful tracial state. It follows that $\mathbb{C} S_{\infty}$ is stably finite as well.

We shall next define a notion of length for elements of $B C * S_{\infty}$. Indeed, each $u \in$ $\left(B C * S_{\infty}\right) \backslash\{e\}$ has a unique expression of the form $w_{1} w_{2} \cdots w_{n}$, for some $n \in \mathbb{N}$ and some $w_{1}, \ldots, w_{n} \in(B C \backslash\{e\}) \cup\left\{t_{j}, t_{j}^{*}: j \in \mathbb{N}\right\}$, satisfying $w_{i+1} \in\left\{t_{j}, t_{j}^{*}: j \in \mathbb{N}\right\}$ whenever $w_{i} \in B C \backslash\{e\}(i=1, \ldots, n-1)$. We then define len $u=n$ for this value of $n$, and set len $e=0$. This also gives a definition of length for elements of $S_{\infty}$ by considering $S_{\infty}$ as a submonoid of $B C * S_{\infty}$ in the natural way. For $m \in \mathbb{N}_{0}$ we set

$$
L_{m}\left(B C * S_{\infty}\right)=\left\{u \in B C * S_{\infty}: \text { len } u \leq m\right\}, \quad L_{m}\left(S_{\infty}\right)=\left\{u \in S_{\infty}: \text { len } u \leq m\right\} .
$$

We now describe our embedding of $\mathbb{C} S_{\infty}$ into $\mathbb{C}\left(B C * S_{\infty}\right)$. By Lemma $2.3, \mathbb{C}\left(B C * S_{\infty}\right)$ has a $\mathrm{C}^{*}$-completion $(A,\|\cdot\|)$. Let $\gamma_{n}=\left(n\left\|\delta_{t_{n}}\right\|\right)^{-1}(n \in \mathbb{N})$ and define elements $a_{n}$ in $\mathbb{C}\left(B C * S_{\infty}\right)$ by $a_{n}=\delta_{p}+\gamma_{n} \delta_{t_{n}}(n \in \mathbb{N})$, so that $a_{n} \rightarrow \delta_{p}$ as $n \rightarrow \infty$. Using the universal property of $S_{\infty}$ we may define a unital *-homomorphism $\varphi: \mathbb{C} S_{\infty} \rightarrow \mathbb{C}\left(B C * S_{\infty}\right)$ by setting $\varphi\left(\delta_{t_{n}}\right)=a_{n}(n \in \mathbb{N})$ and extending to $\mathbb{C} S_{\infty}$. In what follows, given a monoid $S$ and $s \in S, \delta_{s}^{\prime}$ will denote the linear functional on $\mathbb{C} S$ defined by $\left\langle\delta_{t}, \delta_{s}^{\prime}\right\rangle=\mathbb{1}_{s, t}(t \in S)$, where $\mathbb{1}_{s, t}=1$ if $s=t$ and $\mathbb{1}_{s, t}=0$ otherwise.

Lemma 3.2. Let $w \in S_{\infty}$ with len $w=m$. Then

(i) $\varphi\left(\delta_{w}\right) \in \operatorname{span}\left\{\delta_{u}: u \in L_{m}\left(B C * S_{\infty}\right)\right\}$; 
(ii) for each $y \in L_{m}\left(S_{\infty}\right)$ we have

$$
\left\langle\varphi\left(\delta_{y}\right), \delta_{w}^{\prime}\right\rangle \neq 0 \Leftrightarrow y=w .
$$

Proof. We proceed by induction on $m$. When $m=0, w$ is forced to be $e$ and hence, as $\varphi$ is unital, $\varphi\left(\delta_{e}\right)=\delta_{e}$, so that (i) is satisfied. In (ii), $y$ is also equal to $e$, so that (ii) is trivially satisfied as well.

Assume $m \geq 1$ and that (i) and (ii) hold for all elements of $L_{m-1}\left(S_{\infty}\right)$. We can write $w$ as $w=v x$ for some $v \in S_{\infty}$ with len $v=m-1$ and some $x \in\left\{t_{j}, t_{j}^{*}: j \in \mathbb{N}\right\}$.

First consider (i). By the induction hypothesis, we can write $\varphi\left(\delta_{v}\right)=\sum_{u \in E} \alpha_{u} \delta_{u}$, for some finite set $E \subset L_{m-1}\left(B C * S_{\infty}\right)$ and some scalars $\alpha_{u} \in \mathbb{C}(u \in E)$. Suppose that $x=t_{j}$ for some $j \in \mathbb{N}$. Then

$$
\varphi\left(\delta_{w}\right)=\varphi\left(\delta_{v}\right) \varphi\left(\delta_{t_{j}}\right)=\left(\sum_{u \in E} \alpha_{u} \delta_{u}\right)\left(\delta_{p}+\gamma_{j} \delta_{t_{j}}\right)=\sum_{u \in E} \alpha_{u} \delta_{u p}+\alpha_{u} \gamma_{j} \delta_{u t_{j}},
$$

which belongs to $\operatorname{span}\left\{\delta_{u}: u \in L_{m}\left(B C * S_{\infty}\right)\right\}$ because

$$
\operatorname{len}(u p) \leq \operatorname{len}(u)+1 \leq m \text { and } \operatorname{len}\left(u t_{j}\right)=\operatorname{len}(u)+1 \leq m
$$

for each $u \in L_{m-1}\left(B C * S_{\infty}\right)$. The case $x=t_{j}^{*}$ is established analogously.

Next consider (ii). Let $y \in L_{m}\left(S_{\infty}\right)$. If len $y \leq m-1$ then, by (i), we know that $\varphi\left(\delta_{y}\right) \in$ $\operatorname{span}\left\{\delta_{u}: u \in L_{m-1}\left(B C * S_{\infty}\right)\right\} \subset \operatorname{ker} \delta_{w}^{\prime}$. Hence in this case $y \neq w$ and $\left\langle\varphi\left(\delta_{y}\right), \delta_{w}^{\prime}\right\rangle=0$.

Now suppose instead that len $y=m$, and write $y=u z$ for some $u \in L_{m-1}\left(S_{\infty}\right)$ and $z \in\left\{t_{j}, t_{j}^{*}: j \in \mathbb{N}\right\}$. By (i) we may write $\varphi\left(\delta_{u}\right)=\sum_{s \in F} \beta_{s} \delta_{s}$ for some finite subset $F \subset$ $L_{m-1}\left(B C * S_{\infty}\right)$ and some scalars $\beta_{s} \in \mathbb{C}(s \in F)$, and we may assume that $v \in F$ (possibly with $\left.\beta_{v}=0\right)$. We prove the result in the case that $z=t_{j}$ for some $j \in \mathbb{N}$, with the argument for the case $z=t_{j}^{*}$ being almost identical. We have $\varphi\left(\delta_{z}\right)=\delta_{p}+\gamma_{j} \delta_{t_{j}}$ and it follows that

$$
\varphi\left(\delta_{y}\right)=\varphi\left(\delta_{u}\right) \varphi\left(\delta_{z}\right)=\sum_{s \in F} \beta_{s} \delta_{s p}+\beta_{s} \gamma_{j} \delta_{s t_{j}} .
$$

Observe that $s p \neq w$ for each $s \in F$. This is because we either have len $(s p)<m=\operatorname{len}(w)$, or else $s p$ ends in $p$ when considered as a word over the alphabet $\left\{p, p^{*}\right\} \cup\left\{t_{j}, t_{j}^{*}: j \in \mathbb{N}\right\}$, whereas $w \in S_{\infty}$. Moreover, given $s \in F, s t_{j}=w=v x$ if and only if $s=v$ and $t_{j}=x$. Hence

$$
\left\langle\varphi\left(\delta_{y}\right), \delta_{w}^{\prime}\right\rangle=\beta_{v} \gamma_{j} \mathbb{1}_{t_{j}, x}=\left\langle\varphi\left(\delta_{u}\right), \delta_{v}^{\prime}\right\rangle \gamma_{j} \mathbb{1}_{t_{j}, x}
$$

As $\gamma_{j}>0$, this implies that $\left\langle\varphi\left(\delta_{y}\right), \delta_{w}^{\prime}\right\rangle \neq 0$ if and only if $\left\langle\varphi\left(\delta_{u}\right), \delta_{v}^{\prime}\right\rangle \neq 0$ and $t_{j}=x$, which, by the induction hypothesis, occurs if and only if $u=v$ and $t_{j}=x$. This final statement is equivalent to $y=w$.

Corollary 3.3. The map $\varphi$ is injective.

Proof. Assume towards a contradiction that $\sum_{u \in F} \alpha_{u} \delta_{u} \in \operatorname{ker} \varphi$ for some non-empty finite set $F \subset S_{\infty}$ and $\alpha_{u} \in \mathbb{C} \backslash\{0\}(u \in F)$. Take $w \in F$ of maximal length. Then

$$
0=\left\langle\varphi\left(\sum_{u \in F} \alpha_{u} \delta_{u}\right), \delta_{w}^{\prime}\right\rangle=\sum_{u \in F} \alpha_{u}\left\langle\varphi\left(\delta_{u}\right), \delta_{w}^{\prime}\right\rangle=\alpha_{w}\left\langle\varphi\left(\delta_{w}\right), \delta_{w}^{\prime}\right\rangle,
$$

where the final equality follows from Lemma 3.2(ii). That lemma also tells us that $\left\langle\varphi\left(\delta_{w}\right), \delta_{w}^{\prime}\right\rangle \neq$ 0 , forcing $\alpha_{w}=0$, a contradiction.

We can now prove our main theorem. 
Proof of Theorem 1.1. Recall that $(A,\|\cdot\|)$ denotes a $\mathrm{C}^{*}$-completion of $\mathbb{C}\left(B C * S_{\infty}\right)$, which exists by Lemma 2.3, and $A$ is infinite since $\delta_{p}, \delta_{q} \in A$. Let $A_{0} \subset A$ be the image of $\varphi$. Corollary 3.3 implies that $A_{0} \cong \mathbb{C} S_{\infty}$, which is stably finite by Lemma 3.1. Moreover, $\varphi\left(\delta_{t_{n}}\right)=$ $a_{n} \rightarrow \delta_{p}$ as $n \rightarrow \infty$, so that $\delta_{p} \in \overline{A_{0}}$, and we see also that $\delta_{t_{n}}=\frac{1}{\gamma_{n}}\left(a_{n}-\delta_{p}\right) \in \overline{A_{0}}(n \in \mathbb{N})$. The elements $\delta_{p}$ and $\delta_{t_{n}}(n \in \mathbb{N})$ generate $A$ as a $\mathrm{C}^{*}$-algebra, and since $\overline{A_{0}}$ is a $\mathrm{C}^{*}$-subalgebra containing them, we must have $A=\overline{A_{0}}$, which completes the proof.

Acknowledgements. We are grateful to Yemon Choi for several helpful discussions whilst working on this problem. We are also grateful to the referee for his/her useful comments, in particular those concerning the references for Theorem 2.2.

\section{REFERENCES}

[1] D. Avitzour, Free products of C*-algebras, Trans. Amer. Math. Soc. 271 (1982), 423-435.

[2] B. A. Barnes, Representations of the $\ell^{1}$-algebra of an inverse semigroup, Trans. Amer. Math. Soc. 218 (1976), 361-396.

[3] B. A. Barnes and J. Duncan, The Banach algebra $\ell^{1}(S)$, J. Funct. Anal. 18 (1975), 96-113.

[4] B. Blackadar, Notes on the structure of projections in simple $\mathrm{C}^{*}$-algebras (Semesterbericht Funktionalanalysis), Technical Report W82, Universität Tübingen, March 1983.

[5] B. Blackadar, K-Theory for operator algebras. Second edition. Mathematical Sciences Research Institute Publications, 5. Cambridge University Press, Cambridge, 1998.

[6] D. P. Blecher and V. I. Paulsen, Explicit construction of universal operator algebras and applications to polynomial factorization, Proc. Amer. Math. Soc. 112 (1991), 839-850.

[7] Y. Choi, Directly finite algebras of pseudofunctions on locally compact groups, Glasgow Math. J. 57 (2015), 693-707.

[8] N. P. Clarke, A finite but not stably finite C*-algebra, Proc. Amer. Math. Soc. 96 (1986), 85-88.

[9] M. Rørdam, A simple C*-algebra with a finite and an infinite projection, Acta Math. 191 (2003), 109-142.

[10] M. Rørdam, F. Larsen, and N. J. Laustsen, An introduction to K-theory for $C^{*}$-algebras, London Mathematical Society Student Texts 49, Cambridge University Press, Cambridge, 2000.

Niels Jakob Laustsen/Jared T. White, Department of Mathematics and Statistics, LanCaster University, Lancaster LA1 4YF, United Kingdom

E-mail address: n.laustsen@lancaster.ac.uk, j.white6@lancaster.ac.uk 